JOURNAL OF SYNCHROTRON RADIATION

ISSN 1600-5775

\title{
Design simulations of a horizontally deflecting high-heat-load monochromator
}

\author{
Philipp Brumund,* Juan Reyes-Herrera, Carsten Detlefs, Christian Morawe, \\ Manuel Sanchez del Rio and Aleksandr I. Chumakov¥
}

ESRF - The European Synchrotron, 38043 Grenoble Cedex 9, France. *Correspondence e-mail: brumund@esrf.fr

Received 10 July 2020

Accepted 20 October 2020

Edited by A. Momose, Tohoku University, Japan

₹ Also at National Research Center Kurchatov Institute, 123182 Moscow, Russia.

Keywords: X-ray optics; high-heat-load optics; silicon monochromator; finite element analysis; ray-tracing.

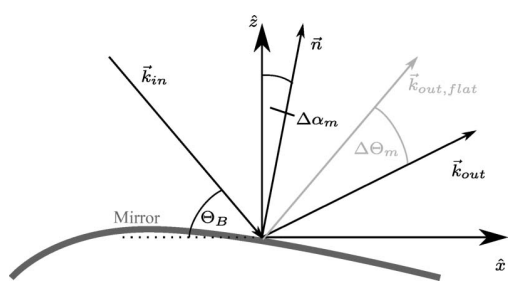

C 2021 International Union of Crystallography
The performance of a liquid-nitrogen-cooled high-heat-load monochromator with a horizontal scattering plane has been analysed, aiming to preserve the high quality of the X-ray beam in the vertical plane for downstream optics. Using finite-element analysis, height profiles of the crystal surface for various heat loads and the corresponding slope errors in the meridional and sagittal planes were calculated. Then the angular distortions of the reflected beam in both meridional and sagittal planes were calculated analytically and also modelled by ray tracing, revealing a good agreement of the two approaches. The results show that with increasing heat load in the crystal the slope errors of the crystal surface reach their smallest values first in the sagittal and then in the meridional plane. For the considered case of interest at a photon energy of $14.412 \mathrm{keV}$ and the $\mathrm{Si}(111)$ reflection with a Bragg angle of $7.88^{\circ}$, the angular distortions of the reflected beam in the sagittal plane are an order of magnitude smaller than in the meridional one. Furthermore, they are smaller than the typical angular size of the beam source at the monochromator position. For a high-heat-load monochromator operating in the horizontal scattering plane, the sagittal angular distortions of the reflected beam appear in the vertical plane. Thus, such an instrument perfectly preserves the high quality of the X-ray beam in the vertical plane for downstream optics. Compared with vertical scattering, the throughput of the monochromatic beam with the horizontal scattering plane is reduced by only $3.3 \%$ for the new EBS source, instead of 34.3\% for the old ESRF-1 machine. This identifies the horizontal-scattering high-heat-load monochromator as a device essentially free of the heat-load effects in the vertical plane and without significant loss in terms of throughput.

\section{Introduction}

In 2020, the European Synchrotron Radiation Facility (ESRF) started exploring new terrain by taking into service the new Extremely Brilliant Source (EBS) machine (Biasci et al., 2014; Raimondi, 2016). The new source provides X-ray beams with much smaller horizontal size and divergence. In addition to tremendously increased brilliance, this also allows for new, more efficient, X-ray optics.

In this paper, we analyse the scheme of a high-heat-load monochromator (HHLM) with horizontal scattering plane, aiming to preserve the high quality of the X-ray beam in the vertical plane for downstream optics. The high quality ${ }^{1}$ of the $\mathrm{X}$-ray beam is important for many synchrotron spectroscopy techniques. For example, for nuclear inelastic scattering and inelastic X-ray scattering, the quality of the wavefront in the vertical plane directly determines the shape of the instrumental function and the energy resolution of phonon spectroscopy (see, for example, Chumakov et al., 2019).

\footnotetext{
${ }^{\mathbf{1}}$ For the purposes of this paper, we define 'high quality' as the absence of any beam perturbation, not considering the possible use of corrective optics.
} 
Table 1

Comparison of the source parameters at a high- $\beta$ straight section of the previous ESRF-1 with the new EBS machine for a photon energy of $14.412 \mathrm{keV}$.

Source size and divergence are calculated considering the horizontally polarized radiation of a $4.8 \mathrm{~m}$-long U20 undulator and following the approach described by Onuki \& Elleaume (2003) and Tanaka \& Kitamura (2009). In timing modes, the vertical source emittance $\epsilon_{\mathrm{v}}$ is enlarged in order to improve the life time. This enlargement does not affect the horizontal and vertical beta functions, $\beta_{\mathrm{h}, \mathrm{v}}$, of the machine lattice.

\begin{tabular}{|c|c|c|c|c|c|}
\hline Source & Direction & $\begin{array}{l}\text { Emittance } \\
\epsilon(\mathrm{pm}) \\
\text { r.m.s. }\end{array}$ & $\begin{array}{l}\text { Beta } \\
\beta(\mathrm{m})\end{array}$ & $\begin{array}{l}\text { Source size } \\
S(\mu \mathrm{m}) \\
\text { FWHM }\end{array}$ & $\begin{array}{l}\text { Divergence } \\
\Delta \theta(\mu \mathrm{rad}) \\
\text { FWHM }\end{array}$ \\
\hline \multicolumn{6}{|c|}{ Multibunch modes } \\
\hline High- $\beta$ & Vertical & 5 & 2.94 & 13.3 & 9.5 \\
\hline High $-\beta$ & Horizontal & 4000 & 37.8 & 968.6 & 25.9 \\
\hline EBS & Vertical & 5 & 2.65 & 12.9 & 9.6 \\
\hline EBS & Horizontal & 150 & 6.90 & 76.5 & 14.2 \\
\hline \multicolumn{6}{|c|}{ Timing modes } \\
\hline High- $\beta$ & Vertical & 50 & 2.94 & 30.2 & 13.3 \\
\hline High- $\beta$ & Horizontal & 4000 & 37.8 & 968.6 & 25.9 \\
\hline EBS & Vertical & 50 & 2.65 & 28.8 & 13.6 \\
\hline EBS & Horizontal & 150 & 6.90 & 76.5 & 14.2 \\
\hline
\end{tabular}

At present storage rings the horizontal emittance is generally larger than the vertical one (see Table 1). Typical undulators use magnet arrays above and below the orbit plane in order to operate at the smallest possible gap (Onuki \& Elleaume, 2003; Chavanne et al., 2011). Unless special magnet structures are used, such devices produce horizontally polarized radiation. Consequently, in many cases, the sensitive downstream optics have to operate in the vertical scattering plane, in order to avoid considerable losses of intensity due to small polarization factors at high scattering angle (e.g. optics for nuclear resonance scattering), due to the worse emittance in the horizontal plane, and due to technical convenience (e.g. inelastic X-ray scattering). Such optics require high quality of the X-ray beam specifically in the vertical plane.

At present, however, the quality of the beam in the vertical plane is degraded by high-heat-load monochromators because they diffract X-rays in the vertical scattering plane (Lee et al., 2000, 2001; Carpentier et al., 2001; Tajiri et al., 2001; Zhang et al., 2003, 2013; Chumakov et al., 2004, 2014). The choice of an HHLM in the vertical scattering plane is natural for facilities where the vertical divergence is considerably smaller than the horizontal one, and therefore much better matches the intrinsic angular acceptances of the HHLM's crystals. For preEBS sources, a horizontal HHLM would deliver a beam with wider bandwidth and reduced throughput (see comparisons of throughputs in Section 2).

However, crystal slope errors in the scattering (meridional) plane affect the X-ray beam much stronger than those in the perpendicular (sagittal) plane. Therefore, with the traditional HHLMs in the vertical scattering plane, the induced large angular distortions in the meridional planes are directly coupled and affect the more important vertical parameters of the beam, whereas the smaller distortions of the beam in the sagittal plane are coupled with the less critical horizontal parameters.
The new EBS storage ring provides a beam with a horizontal divergence that is almost as small as the vertical one, especially when operated in timing modes (see Table 1$)^{\mathbf{2}}$, and smaller than the angular acceptances of HHLMs (which are of the order of $20 \mu \mathrm{rad}$ for $\mathrm{Si}(111)$ at $14.412 \mathrm{keV}$. Therefore, there is potential interest in switching HHLMs from diffraction in the vertical to the horizontal scattering plane, because then the most significant angular distortions induced by the HHML are decoupled from the vertical plane. Our analysis is performed specifically for the Nuclear Resonance beamline (Rüffer \& Chumakov, 1996) ID18 of the ESRF. The source is composed of three undulators with period of $20 \mathrm{~mm}$, total length of $4.8 \mathrm{~m}$, and magnetic gap of $11 \mathrm{~mm}$. The undulators provide maximum spectral density at the energy of $14.412 \mathrm{keV}$ in the fundamental.

The slope error of the surface of an optical element (mirror or crystal) $\Delta \alpha$ produces the angular distortion of the reflected beam $\Delta \theta$, but this effect is different in the scattering (meridional) and in perpendicular (sagittal) planes. The distortions are smaller in the sagittal plane as expected from the known relations between the slope errors of the crystal surface and the corresponding angular distortions of the reflected $\mathrm{X}$-ray beam,

$$
\begin{gathered}
\Delta \theta_{\mathrm{m}}=2 \Delta \alpha_{\mathrm{m}}, \\
\Delta \theta_{\mathrm{s}}=2 \Delta \alpha_{\mathrm{s}} \sin \left(\theta_{\mathrm{B}}\right) .
\end{gathered}
$$

Here, $\Delta \alpha_{\mathrm{m}}$ and $\Delta \alpha_{\mathrm{s}}$ are the meridional and the sagittal slope errors of the crystal surface, respectively; $\Delta \theta_{\mathrm{m}}$ and $\Delta \theta_{\mathrm{s}}$ are the corresponding meridional and the sagittal angular distortions of the reflected beam, and $\theta_{\mathrm{B}}$ is the Bragg angle. The effect of sagittal slope errors $\Delta \alpha_{\mathrm{s}}$ is reduced by the 'forgiveness factor' $\sin \left(\theta_{\mathrm{B}}\right)$, as only the component of the wavevector perpendicular to the mirror surface is tilted (DiGennaro et al., 1988; de Castro \& Reininger, 1991). These relations can be obtained using an analytical approach (Appendix $A$ ) or from geometrical analysis (Appendix $B$ ).

For hard X-rays and low-index reflections, the Bragg angle $\theta_{\mathrm{B}}$ is small. Therefore, even for equal slope errors in the two directions $\Delta \alpha_{\mathrm{m}}=\Delta \alpha_{\mathrm{s}}$, the ratio between the meridional and sagittal angular distortions $\Delta \theta_{\mathrm{m}} / \Delta \theta_{\mathrm{s}}=1 / \sin \theta_{\mathrm{B}}$ can be rather large, by an order of magnitude or so (in our case $\theta_{\mathrm{B}}=7.88^{\circ}$, $1 / \sin \theta_{\mathrm{B}}=7.3$ ). This holds for all slope errors of the crystal, e.g. caused by heat load, static deformations, vibrations, etc. Typical grazing angles for mirrors or multilayer monochromators are even smaller, such that the effect is even more pronounced.

In this paper, we first compare the throughput of monochromators with horizontal and vertical scattering plane for both former ESRF-1 and new EBS machines (Section 2), assuming horizontally polarized undulator radiation. We then analyse specifically the meridional and sagittal angular distortions of the reflected beam caused by heat-load effects

\footnotetext{
${ }^{2}$ Here and below, all source and beam parameters, slope errors and angular distortions of the reflected beam are given as full widths at half-maximum (FWHM), except for emittances, which are given as root mean squares (r.m.s.).
} 
(Section 3). The results are discussed in Section 4 and summarized in Section 5.

\section{Throughput of a monochromator in the horizontal and vertical scattering plane}

The goal of this section is to compare the performances of the ideal HHLM (without any deformation) for the different geometrical configurations (horizontal and vertical scattering planes) and for the different sources (ESRF-1 high- $\beta$ and EBS).

Ray-tracing simulations were performed using $S H A D O W$ (Sanchez del Rio et al., 2011) with the interface ShadowOui (Rebuffi \& Sanchez del Rio, 2016). Reflectivity curves were obtained from XOPPY, the Python version of XOP (Sanchez del Rio \& Dejus, 2011; Sanchez del Rio et al., 2015). Both modules are included in the OASYS suite (Rebuffi \& Sanchez del Rio, 2017).

We consider a double-crystal monochromator with two symmetrically cut $\mathrm{Si}(111)$ crystals. Ray-tracing simulations were performed to evaluate the throughput. Depending on the experimental needs, two cases of interest can be identified:

(i) Applications that require a high total intensity, and where monochromatization down to the typical energy bandwidths of the $\mathrm{Si}(111)$ reflection is sufficient [see Fig. 1 for intrinsic energy distributions of the reflected photons for the $\mathrm{Si}(111)$ double-crystal monochromator]. For these cases, the throughput of the total intensity $T_{\mathrm{I}}$ is the relevant parameter. Here, the ray-tracing simulations were performed with a wide source bandwidth.

(ii) Applications that involve high-energy-resolution downstream optics with much smaller bandwidth, and require high spectral density (number of photons per energy interval). In these cases, the peak throughput of monochromatic radiation $T_{\mathrm{M}}$ is most relevant. Here, the ray-tracing simula-

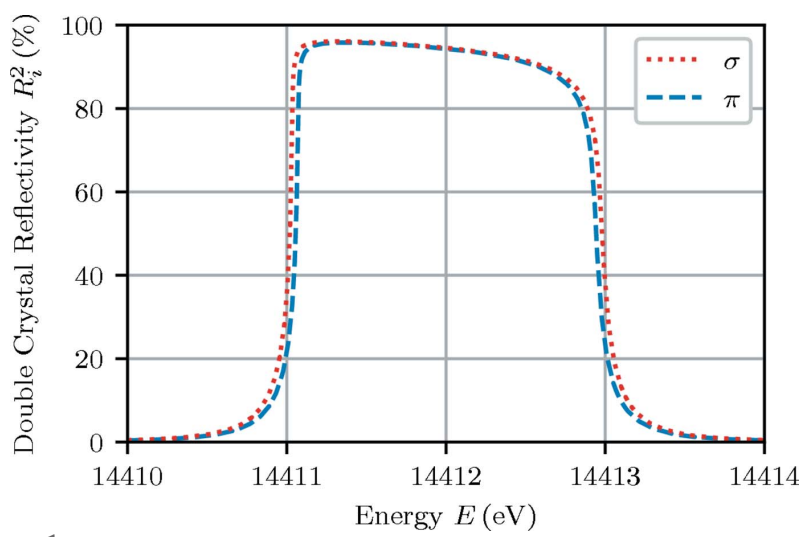

Figure 1

Intrinsic (i.e. for zero angular divergence of incident radiation) reflectivity of a $\mathrm{Si}(111)$ double-crystal monochromator at $14.412 \mathrm{keV}$ for $\sigma$ - and $\pi$-polarized radiation. The peak reflectivity is nearly identical, and the polarization factor affects mainly the energy bandwidth, via the difference in the angular Darwin width. The throughputs for $\sigma$ - and $\pi$ polarized radiation correspond to diffraction in the vertical and horizontal scattering plane, respectively, and their corresponding energy bandwidths are $1.966 \mathrm{eV}$ and $1.892 \mathrm{eV}$ or, in angular units, $18.3 \mu \mathrm{rad}$ and $17.7 \mu \mathrm{rad}$.
Table 2

Throughputs of a $\mathrm{Si}(111)$ double-crystal monochromator for diffraction in the horizontal and vertical scattering plane.

Throughputs are stated as relative values in percentage of maximum value per column. For both scattering planes, we present the results for a high- $\beta$ section of the previous ESRF source and the new ESRF-EBS machine. For general applications, monochromatization down to the typical bandwidth of the $\mathrm{Si}(111)$ reflection is sufficient. In this case, the energy bandwidth $\Delta E$ and the throughput of total intensity $T_{\mathrm{I}}$ of the reflected radiation are the relevant parameters. High-resolution techniques as employed on ID18, however, use narrow energy bandwidths and, thus, require high spectral density. In this case, the throughput of monochromatic radiation $T_{\mathrm{M}}$ is the relevant parameter to optimize.

\begin{tabular}{lllll}
\hline & Scattering & Bandwidth & Total flux & Peak intensity \\
Source & plane & $\Delta E$ (FWHM) & $T_{\mathrm{I}}$ & $T_{\mathrm{M}}$ \\
\hline
\end{tabular}

Multibunch mode $\left(\epsilon_{\mathrm{v}}=5 \mathrm{pm}\right)$

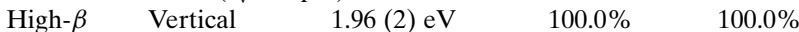

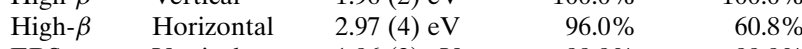

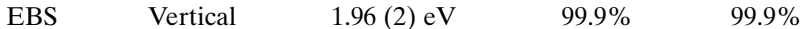

$\begin{array}{lll}\text { EBS Horizontal } & 2.08(2) \mathrm{eV} & 95.9 \%\end{array}$

Timing modes $\left(\epsilon_{\mathrm{v}}=50 \mathrm{pm}\right)$

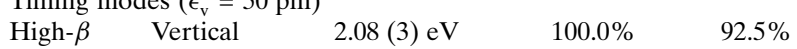

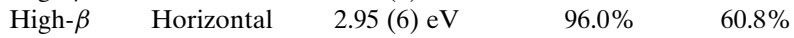

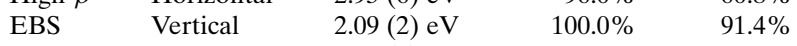

$\begin{array}{llll}\text { EBS Horizontal } & 2.07(3) \mathrm{eV} & 95.9 \% & 88.4 \%\end{array}$

tions were performed with a zero source bandwidth, i.e. with a perfectly monochromatic source.

To account for all possible cases, a total of 16 ray-tracing simulations were carried out, combining the following options:

(i) Source: new EBS or old ESRF-1 (high- $\beta$ straight section).

(ii) Machine mode: multibunch mode $\left(\epsilon_{\mathrm{v}}=5 \mathrm{pm}\right)$ or timing mode $\left(\epsilon_{\mathrm{v}}=50 \mathrm{pm}\right)$.

(iv) Considered source bandwidth: wide or zero.

(iii) Scattering plane: horizontal or vertical.

The throughput was derived by ray-tracing simulations performed with Gaussian profiles for the source size and divergence. In each simulation, the average and standard deviation of 20 repetitions with one million rays were calculated. For calculations of the throughput of total intensity $T_{\mathrm{I}}$ we used a bandwidth of $6 \mathrm{eV}$ around the central beam energy $14.142 \mathrm{keV}$, whereas, for calculations of the peak throughput of monochromatic radiation $T_{\mathrm{M}}$, the bandwidth was set to zero. After double reflection on two flat $\mathrm{Si}(111)$ crystals, the ray intensity was obtained, as presented in Table 2 . The calculated throughputs of total intensity $T_{\mathrm{I}}$ and monochromatic radiation $T_{\mathrm{M}}$ are presented in percent, with $100 \%$ being the reference of the highest value for each calculated throughput. Intensity profiles versus photon energy were also calculated. The width of these energy distributions (FWHMs) are reported as the energy bandwidth in Table 2.

Table 2 compares the throughput of a double-crystal monochromator with horizontal and vertical scattering plane for a high- $\beta$ section at the previous ESRF-1 with the new EBS machine.

For both sources, the total transmitted intensity (throughput of total intensity $T_{I}$ ) is similar. The horizontal scattering plane is only slightly disadvantageous as $T_{\mathrm{I}}$ is reduced by the polarization factor, $P=\cos \left(2 \theta_{\mathrm{B}}\right) \simeq 96.2 \%$, see Fig. 1 . The exact 
value of $T_{\mathrm{I}}$ is $96.0 \%$, i.e. less than $P$, as, in addition to the decrease of the reflection bandwidth for each plane wave by a factor of $P$ (Fig. 1), the polarization factor also slightly decreases the reflectivity.

The divergence of the source affects the bandwidth of the reflected beam and the spectral density (throughput of monochromatic radiation $T_{M}$ ). Therefore, similar values are obtained for ESRF-1 and EBS in the vertical scattering plane, where the divergence is practically identical before and after the machine upgrade. The bandwidths of reflected radiation for the vertical scattering plane are close to the intrinsic $\mathrm{Si}(111)$ bandwidth of $1.966 \mathrm{eV}$, in particular for machine modes with small vertical divergence $\left(\Delta \theta_{\mathrm{v}}=9.5 \mu \mathrm{rad}\right)$. The relatively high horizontal divergence of the old machine (we consider only high- $\beta$ sections) of about $\Delta \theta_{\mathrm{h}}=25.9 \mu \mathrm{rad}$, however, leads to a significant increase in the reflected bandwidth of up to 2.97 (4) $\mathrm{eV}$.

The throughput of monochromatic radiation $T_{\mathrm{M}}$ in horizontal scattering is quite low (60.8\%) for the ESRF-1 source, therefore unacceptable for high-resolution applications as relevant for ID18 that are inherently flux limited. For the ESRF-EBS source, the horizontal and vertical divergences are close. For multibunch modes, the loss of peak intensity $T_{\mathrm{M}}$ upon switching from vertical to horizontal scattering is reduced to $\sim 11.5 \%$ for the new EBS machine, compared with $\sim 39.2 \%$ for the old machine ESRF-1. For timing modes, the horizontal and vertical divergences are almost identical: switching to the horizontal scattering plane results in the loss of a peak intensity $T_{\mathrm{M}}$ of only $\sim 3.3 \%$.

\section{Surface deformation under thermal load and its effect on the diffracted beam}

The calculations of the surface deformation under thermal load were performed for an HHLM with vertical scattering plane. However, as discussed below, the distribution of the thermal load over the crystal surface and, therefore, also the surface deformation are practically identical for the cases of the vertical and horizontal scattering planes. Therefore, the results presented below can be used to evaluate the performance of the high-heat-load monochromator both with the vertical and horizontal scattering plane. For the HHLM with the vertical scattering plane, the calculated meridional angular distortions describe the beam properties in the vertical plane, whereas the sagittal distortions are related to the beam properties in the horizontal plane. For the HHLM with the horizontal scattering plane it is the other way around: the sagittal angular distortions describe the beam properties in the vertical plane, and the meridional distortion describes the beam properties in the horizontal plane.

We use finite-element analysis (FEA) to calculate the deformation of the crystal surface for various heat loads obtained by varying the electron ring current. For the FEA simulations we used the commercial software ANSYS (http:// ansys.com). The results of the FEA show a deformed 2D surface. The 2D distribution of slopes in the meridional and sagittal directions are obtained from derivatives of the surface
Table 3

Possible beam sizes of white and monochromatic beam at the position of the monochromator ( $31 \mathrm{~m}$ from the source) when ignoring the $2 \mathrm{~mm} \times$ $1 \mathrm{~mm}$ aperture of the upstream primary slit ( $28 \mathrm{~m}$ from the source).

The values are reported in FWHMs, for the old and new ESRF machine in timing modes, for horizontal $(\mathrm{H})$ and vertical $(\mathrm{V})$ directions. The white beam dimensions describe the power density distribution, the monochromatic beam size is given for X-rays with the energy of $14.412 \mathrm{keV}$. In practical use, the dimensions of the white beam for both machines are truncated by the upstream primary slit to $2.2 \mathrm{~mm} \times 1.1 \mathrm{~mm}$ (see text), whereas the FWHMs of the monochromatic beam are not affected.

\begin{tabular}{llllll}
\hline & \multicolumn{2}{l}{ White beam } & & \multicolumn{2}{l}{ Monochromatic beam } \\
\cline { 2 - 3 } Source & $\mathrm{H}(\mathrm{mm})$ & $\mathrm{V}(\mathrm{mm})$ & & $\mathrm{H}(\mathrm{mm})$ & $\mathrm{V}(\mathrm{mm})$ \\
\hline High- $\beta$ & 2.87 & 2.73 & & 1.26 & 0.41 \\
EBS & 2.71 & 2.52 & & 0.44 & 0.42 \\
\hline
\end{tabular}

profile. The 'weighted' slope errors $\Delta \alpha_{\mathrm{m}, \mathrm{s}}$ are obtained by calculating the respective standard deviations of these distributions after being weighted with the beam intensity (see Section 3.2). Then the angular distortions of the reflected beam $\Delta \theta_{\mathrm{m}, \mathrm{s}}$ for a crystal with vertical scattering plane are obtained in two ways: (i) analytically from the slope errors, using equations (1) and (2) and (ii) by Monte Carlo raytracing using the initially calculated height profiles of the crystal surface. From these results, conclusions are drawn for a crystal with horizontal scattering plane, attributing the sagittal angular distortions $\Delta \theta_{\mathrm{s}}$ to properties of the beam in the vertical plane, and the meridional distortion $\Delta \theta_{\mathrm{m}}$ to beam properties in the horizontal plane.

\subsection{Thermo-mechanical modelling with FEA}

The height profiles of the crystal surface were calculated for various heat loads, resulting from the variation of the storage ring current.

The considered insertion device configuration for ID18 consists of three U20 undulators with deflection parameter $K=$ 0.63 , magnetic period of $20 \mathrm{~mm}$, with 240 periods for all three undulators. The total emitted power for these three undulators is up to $2.5 \mathrm{~kW}$ (electron energy $6 \mathrm{GeV}$, and storage ring current $200 \mathrm{~mA}$ ). At the position of the HHLM located at $31 \mathrm{~m}$ from the centre of the straight section, the on-axis power density would be up to $208 \mathrm{~W} \mathrm{~mm}^{-2}$ (for storage ring current $200 \mathrm{~mA}$ ). Taking into account $8.2 \%$ absorption in an upstream CVD diamond window with a thickness of $300 \mu \mathrm{m}$, the actual peak power density at the position of the HHLM is $191 \mathrm{~W} \mathrm{~mm}^{-2}$. For the FEA simulations the power density distribution was calculated with the $X O P$ code (Sanchez del Rio \& Dejus, 2011) and directly imported as a tabular thermal load with FWHMs of $2.7 \mathrm{~mm} \times 2.5 \mathrm{~mm}$ (EBS machine) if approximated by Gaussian distributions. ${ }^{3}$

The power density distribution does not change significantly between ESRF-1 and EBS machines (see white beam dimensions in Table 3), hence the deformation results are comparable for both machines and only one set for the EBS parameters was calculated. The important point is, however,

${ }^{3}$ Here and below all values are referred to as (horizontal $\times$ vertical). 
that the monochromatic horizontal beam size is reduced for the EBS machine (Table 3), as discussed below.

Ignoring for a moment the aperture of the upstream highpower primary slit $(2.0 \mathrm{~mm} \times 1.0 \mathrm{~mm}, 28 \mathrm{~m}$ from the centre of the straight section), the beam dimensions for the white beam and monochromatic beam at the position of the HHLM ( $31 \mathrm{~m}$ from the centre of the straight section) are stated in Table 3. For both old ESRF-1 and new EBS machines, the horizontal and vertical FWHMs of the white beam are close to each other, revealing approximately axially symmetric power density. Furthermore, the FWHMs of the density distribution are bigger then the projected aperture of the primary slit at the HHLM position (about $2.2 \mathrm{~mm} \times 1.1 \mathrm{~mm}$ for the HHLM with vertical scattering plane). Therefore, the distribution of the power density over the silicon crystal surface is determined mainly by the aperture of the primary slit, and it is practically identical for the HHLM in the vertical scattering plane with $2 \mathrm{~mm} \times 1 \mathrm{~mm}$ slit and for HHLM in the horizontal scattering plane with a rotated $1 \mathrm{~mm} \times 2 \mathrm{~mm}$ slit aperture.

This symmetry is exploited in the FEA calculations, allowing to reuse the same simulation results for both scattering geometries. As discussed above, for the HHLM with vertical scattering plane and $2 \mathrm{~mm} \times 1 \mathrm{~mm}$ aperture of the primary slit, the calculated meridional angular distortions describe the beam properties in the vertical plane, whereas the sagittal distortion are related to the beam properties in the horizontal plane. For the HHLM with the horizontal scattering plane and $1 \mathrm{~mm} \times 2 \mathrm{~mm}$ slit aperture, the calculated sagittal angular distortions describe the beam properties in the vertical plane, and the meridional distortion describes the beam properties in the horizontal plane. Note that the chosen opening of the primary slit corresponds to the optimal conditions of the currently employed high-heat-load monochromator at ID18 (Chumakov et al., 2014) for the storage ring current of $200 \mathrm{~mA}$.

We consider a symmetric $\mathrm{Si}(111)$ reflection. For X-rays with of energy $14.412 \mathrm{keV}$, this corresponds to an incidence angle of $\theta_{\mathrm{B}}=7.88^{\circ}\left[\sin \left(\theta_{\mathrm{B}}\right)=0.137\right]$, giving the iluminated area of the crystal of $2.2 \mathrm{~mm}$ perpendicular to the beam by $8.0 \mathrm{~mm}$ along the beam. The crystal is illuminated on its top face centre, leading to a symmetrical load situation considering both the longitudinal and the transverse plane.

The FEA calculations were performed for the actual size of the silicon crystal at ID18, i.e. $80 \mathrm{~mm}$ (length) $\times 30 \mathrm{~mm}$ (width) $\times 40 \mathrm{~mm}$ (height). A detailed overview of the cooling assembly is given by Chumakov et al. (2014). Both side-cooled surfaces were assumed to be cooled by a liquid-nitrogen cooler clamped to the crystal sides with constant clamping pressure distribution over the surfaces. Under such conditions the coolers can be modelled using a constant convective cooling coefficient on the crystal sides, which we assume to be $3.0 \mathrm{~kW} \mathrm{~m}^{-2} \mathrm{~K}^{-1}$ with the reference temperature $77 \mathrm{~K}$ (liquid nitrogen). This value was confirmed with experimental tests of the same crystal cooling setup (Chumakov et al., 2014).

The beam footprint was meshed with a controlled mesh size of $0.1 \mathrm{~mm}$ element edge length. This mesh size was validated with a mesh study. Decreasing the mesh size further does not change the relevant temperature and displacement results significantly (changes $<1 \%$ ).

We used the temperature-dependent isotropic thermal conductivity (Touloukian et al., 1971) and thermal expansion (Middelmann et al., 2015) shown in Fig. 2. The anisotropy of silicon elasticity was taken into account (Zhang et al., 2014). However, no further attention was paid to the crystal orientation and the material properties of a $\mathrm{Si}(100)$ crystal were used. The influence of this decision on calculated thermal slope errors is predicted in the cited reference to be of maximum $8.1 \%$. A test calculation with $\mathrm{Si}(111)$ properties revealed the error to be $\sim 5 \%$.

For the thermal FEA calculations we assumed that the thermal heat load absorbed on the crystal surface amounts to $90 \%$ of the incident power. The remaining $10 \%$ is considered as a single correction for all possible energy loses. Possible deviations of the assumed correction from an exact value are not important, as all results below are discussed primarily in terms of the absorbed power, whereas the storage ring current is given mainly for reference.

The reliability of the thermal FEA calculations was verified by calculating the temperature in the centre of the upstream face of the crystal for various ring currents. The obtained temperature evolution of this point with increasing heat load was found to be in excellent agreement with the experimental data [Fig. 4 of Chumakov et al. (2014)].

From FEA, we extracted the height profiles and then calculated the slope profiles (height profile derivatives) in both meridional and sagittal directions. From the slope profiles, one can calculate statistically meaningful values such as mean, root mean square (r.m.s.) or standard deviation of slope errors over the whole illuminated beam footprint. Due to the narrower profile of the monochromatic reflected beam, however, slope errors far from the beam centre are less relevant when calculating distortions of the X-ray beam. In order to correctly estimate a value of slope error, one has to weight them with an appropriate weighting function (see Appendix $C$ ), which is different for the previous ESRF-1 and the new ESRF-EBS source.

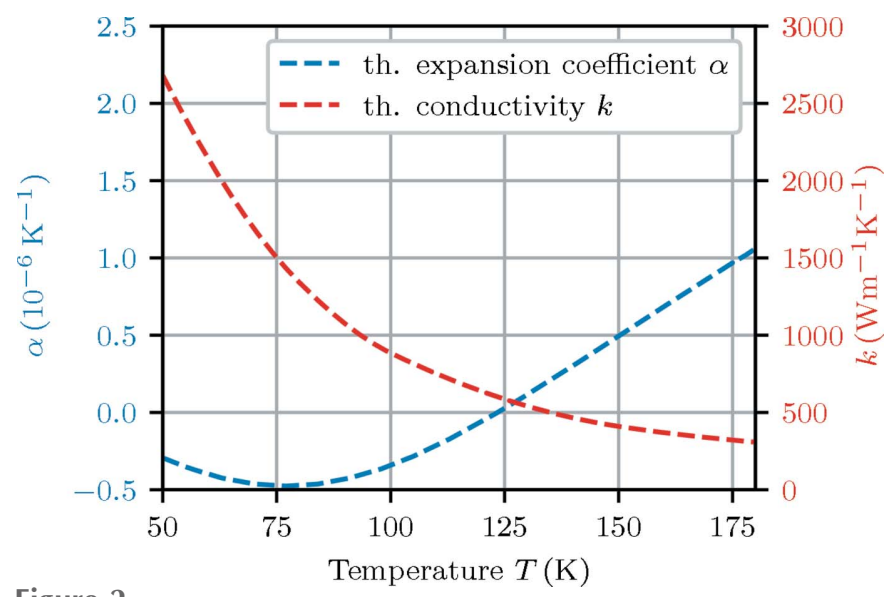

Figure 2

Temperature-dependent thermal expansion $\alpha(T)$ and thermal conductivity $k(T)$ of silicon used for this work. 
In the following, all values for slope errors and angular distortions are stated as FWHMs. They were determined via calculation of standard deviations $\sigma$ and conversion (FWHM = $2.355 \sigma$ ) of the respective considered distributions. This choice was made for easier comparison of the obtained results to available experimental data. The centred heat load on the crystal top face leads to symmetrical surface shapes in the longitudinal and transverse plane. Thus, due to this symmetry, the mean slope is zero and the r.m.s. and standard deviation $\sigma$ for these distributions are identical.

\subsection{Calculations of the angular distortions of the reflected beam}

Here, as in the calculation of the HHLM throughputs, we used the package SHADOW (Sanchez del Rio et al., 2011) with the ShadowOUI interface (Rebuffi \& Sanchez del Rio, 2016), available as a module of the OASYS suite (Rebuffi \& Sanchez del Rio, 2017).

In order to distinguish the small angular distortions of the reflected beam induced by the slope errors of the crystal surface from the larger beam divergence, the incident beam used for the ray-tracing simulations was assumed fully collimated, with the spatial profiles given by the considered central cones of monochromatic radiation. In order to model the diffraction of X-rays on the disturbed HHLM crystal, we simulated this process by rays reflecting on a plane mirror with $100 \%$ reflectivity and a grazing angle of $7.88^{\circ}$, corresponding to the Bragg angle of $14.412 \mathrm{keV}$ radiation for the $\mathrm{Si}(111)$ reflection. The plane surface profile was modified to account for the calculated crystal surface deformation. Finally, we retrieved the statistics of rays downstream from the mirror reflection by computing the intensity distributions versus meridional and sagittal angles and retrieving the FWHM. One single bounce has been considered, because the second crystal is assumed to be flat, i.e. without surface deformations from thermal loads.

For each surface height profile obtained for a given heat load, we performed ten simulations using 400000 rays each. In each simulation, rays were generated by a randomly seeded Monte Carlo routine that samples the source characteristics. The results obtained for each heat-load condition are reported as the average of the ten runs. The standard deviation of the results was about $0.1 \%$ of the average.

\subsection{Results}

The deformed crystal surface shapes for different absorbed powers obtained from FEA calculations are shown in Fig. 3 for the beam footprint area. When increasing the deposited power on the crystal, we observe an evolution from a thermal-dent to a thermal-bump shape with two intermediate patterns discussed below. The surface coordinates $x$ and $y$ lie in the meridional direction (in the scattering plane) and in the perpendicular sagittal direction, respectively. The origin $(0,0,0)$ corresponds to the centre of the crystal surface (before deformation) and the centre of the beam footprint. The height

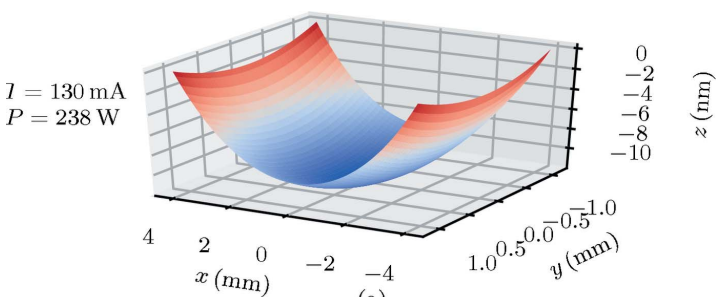

(a)

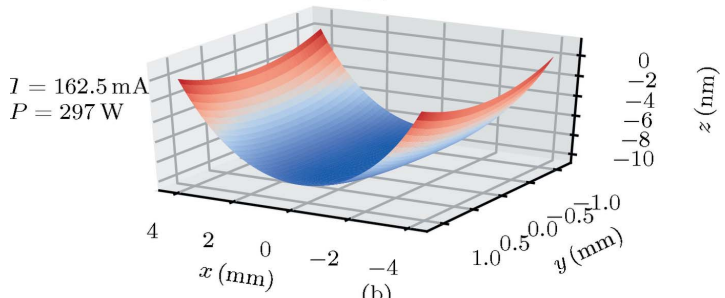

(b)

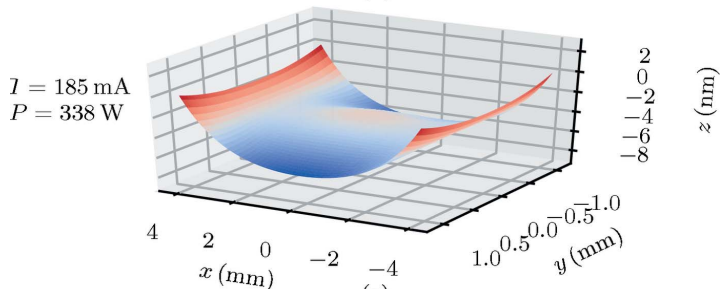

(c)

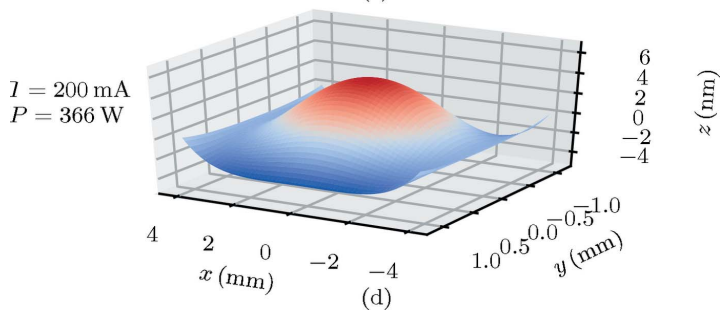

Figure 3

Evolution of the silicon crystal surface (within the white beam footprint area) at increasing heat load from $(a)$ thermal-dent to $(d)$ thermal-bump shape, with two most flat intermediate shapes corresponding to $(b)$ smallest sagittal and $(c)$ meridional slope errors (see text). The corresponding storage ring currents and absorbed powers are stated. The surface coordinates $x$ and $y$ lie in the meridional direction (in the scattering plane) and in the perpendicular sagittal direction, respectively. The calculated heigh profiles are valid for both ESRF-1 and EBS machines since the heat load does not change significantly.

$z$ is the deviation of the surface from a flat plane, $z$-positive pointing outwards.

The evolution of the slope errors (given as FWHM) with increasing heat load in the meridional and sagittal directions is shown in Fig. 4. Here, the slope errors are calculated over the entire area of the crystal surface illuminated by the white beam, along the centre lines in both the meridional (along $y=0$ ) and sagittal direction (along $x=0$ ). With increasing absorbed power (top $x$-axis), both slope errors first increase, revealing nearly the same values. In the power range 200 $240 \mathrm{~W}$, they reach local maxima. Here the crystal surface shows the pronounced thermal-dent shape corresponding to Fig. 3(a). With further increase of power, the slope errors decrease, first in the sagittal and later in the meridional direction. The smallest slope errors are reached first in the sagittal direction, at a power of about $300 \mathrm{~W}$. This corresponds to the height profile shown in Fig. 3(b). At about $340 \mathrm{~W}$, the 
smallest slope error in the meridional direction occurs. This corresponds to the crystal shape shown in Fig. 3(c). The values of the smallest sagittal and meridional errors in two corresponding minima are close to each other, both about $2 \mu \mathrm{rad}$ (FWHM). With further increase of the heat load, both slope errors grow rapidly, indicating the thermal-bump shape of the crystal surface [Fig. 3(d)].

In order to evaluate the angular distortions of the monochromatic $\mathrm{X}$-ray beam reflected by the crystal using equations (1) and (2), the slope errors of the entire illuminated surface have to be weighted by the spatial distribution of monochromatic $14.412 \mathrm{keV}$ photons, which is concentrated within the narrower central cone. This is done using equation (27) and the procedure described in the corresponding section of Appendix $C$.

The weighted slope errors were calculated for two sets of the central cone parameters. In the first case [Fig. 5(a)], we used the parameters of the previous ESRF-1 machine, in order to compare the obtained results with the available experimental data. In the second case [Fig. 5(b)], we used the parameters of the new ESRF-EBS machine, in order to estimate the future performance of a high-heat-load monochromator with the new source. ${ }^{4}$ For both machines, the minima of the weighted slope errors are also compared numerically in Table 4.

The horizontal and vertical sizes of the monochromatic beam central cones for both machines are given in Table 3. For the previous ESRF-1 machine, the central cone of the reflected $14.412 \mathrm{keV} \mathrm{X}$-rays at the position of the high-heatload monochromator is described by the two-dimensional Gaussian distribution with the characteristic widths (FWHM) of $1.26 \mathrm{~mm} \times 0.41 \mathrm{~mm}$. This corresponds to the footprint on the crystal surface of $1.26 \mathrm{~mm}$ perpendicular to the beam and $3.0 \mathrm{~mm}$ along the beam, considerably smaller than the footprint of white radiation $(2.22 \mathrm{~mm} \times 7.95 \mathrm{~mm})$. For the new EBS machine, the central cone is greatly reduced in the horizontal direction, giving a circular cone with the widths ${ }^{5}$ (FWHM) of $0.44 \mathrm{~mm} \times 0.42 \mathrm{~mm}$. This corresponds to the yet smaller footprint of $0.44 \mathrm{~mm}$ perpendicular to the beam and $3.1 \mathrm{~mm}$ along the beam.

The weighted slope errors calculated from the simulated deformations of the crystal surface are shown in Figs. 5(a) and 5(b) for ESRF-1 and ESRF-EBS, respectively. The corresponding angular distortions of the monochromatic reflected beam are shown in Figs. 5(c) and 5(d). As discussed above (Section 3.1), for a high-heat-load monochromator with horizontal scattering plane, the calculated sagittal angular

\footnotetext{
${ }^{4}$ The spatial distribution of the heat load and, accordingly, the deformations of the entire crystal surface are practically identical for the two machines.

${ }^{5}$ This is valid for timing modes of the storage ring operation, with the vertical emittance of $50 \mathrm{pm}$.
}

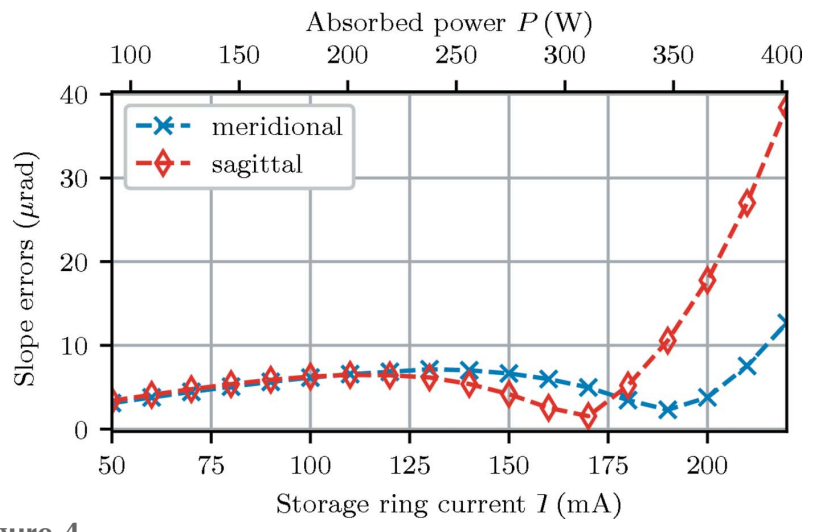

Figure 4

Evolution of the meridional and sagittal slope errors (FWHM) of the entire area of the crystal surface illuminated by white beam with increasing absorbed power of the monochromator crystal $P$ and directly linked storage ring current $I$. Slope errors are calculated as the widths of the distributions $\Delta \alpha_{\mathrm{m}}(x)=\partial z(x, y) /\left.\partial x\right|_{y=0}$ and $\Delta \alpha_{\mathrm{s}}(y)=\partial z(x, y) /\left.\partial y\right|_{x=0}$, respectively, thus along the centre lines in both directions.

distortions correspond to the parameters of the reflected beam in the vertical plane.

\section{Discussion}

The evolution of the thermal deformation measured in terms of slope error follows a kind of general trend (Figs. 4 and 5): at zero power absorbed the crystal is undeformed and the thermal slope errors are zero. When power is added, a concave curvature develops because the coefficient of thermal expansion of $\mathrm{Si}$ is negative at liquid-nitrogen temperature (see Fig. 2). As the power and the temperature increase, the coefficient of thermal expansion becomes positive, eventually reversing the curvature in the centre of the beam spot (Zhang et al., 2013; Chumakov et al., 2014), see Fig. 3. This decreases the slope error until a local minimum is reached. Further increasing the power leads to a convex shape with rapidly increasing slope error (Fig. 4). The optimum working point for our application is at this local minimum.

\subsection{Slope errors}

The crystal shapes shown in Fig. 3 and corresponding slope errors shown in Fig. 4 are calculated for an illumination 
ESRF-1

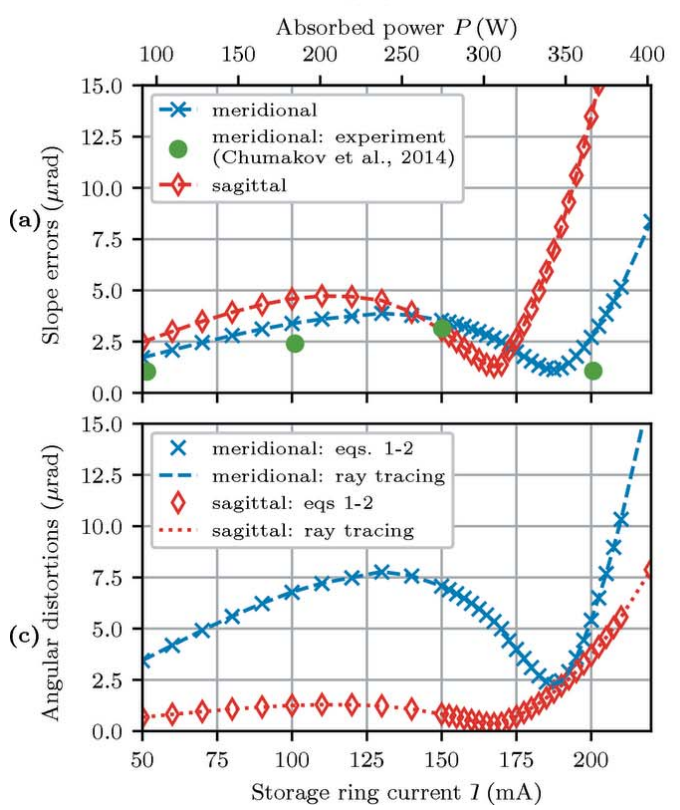

ESRF-EBS

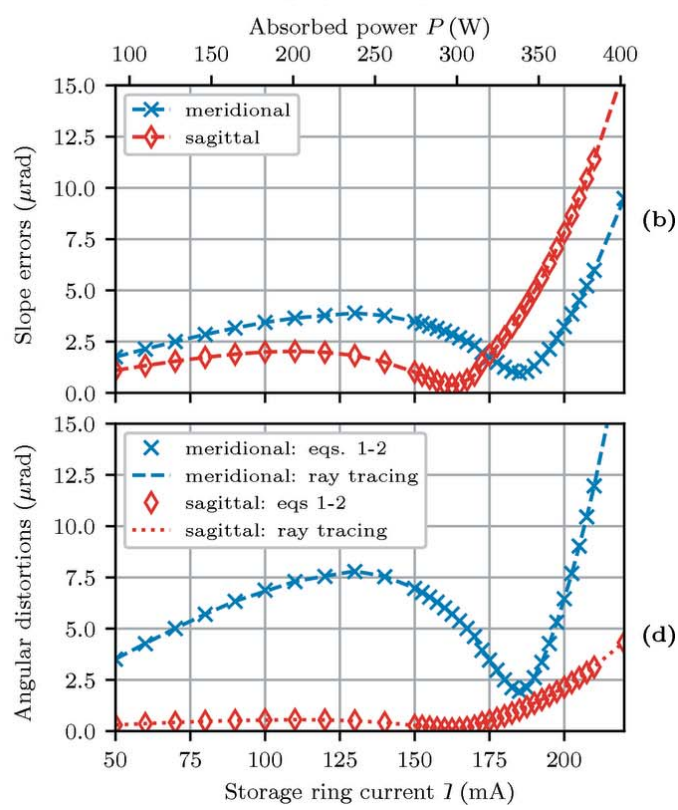

Figure 5

The weighted slope errors of the crystal surface $(a, b)$ and the corresponding angular distortions of the reflected monochromatic beam $(c, d)$ for the previous ESRF-1 $(a, c)$ and the new EBS $(b, d)$ machines in meridional and sagittal planes. All values are given as FWHMs. The calculated slope errors of the crystal surface are weighted by the spatial distribution of the central cone of monochromatic $14.412 \mathrm{keV}$ X-rays. The angular distortions of the reflected beam are calculated from the weighted slope errors using equations (1) and (2) (symbols) and by ray tracing using the simulated height profiles of the crystal surface (lines). For a high-heat-load monochromator with horizontal scattering plane, the calculated sagittal angular distortions correspond to the parameters of the reflected beam in the vertical plane (see text).

condition that, as discussed before, is valid for both ESRF-1 and EBS sources, and for diffraction both in the vertical and horizontal scattering planes. The slope errors are calculated from the normal displacement along the centre axes of the beam footprint on the crystal surface. However, the illuminated area of the 'good photons' (the photons around $14.412 \mathrm{keV}$, close to the experimental condition) is much less than the footprint of the deposited power, and it is different for the ESRF-1 and EBS machines. Therefore, the curve in Fig. 4 (valid for the footprint of white beam) has to be adapted for the different footprints of the monochromatic beam of these machines.

Fig. 5 compares the weighted slope errors of the crystal surface and angular distortion of the reflected radiation calculated for two different machines: ESRF-1 and EBS. The weighted slope errors for ESRF-1 [Fig. 5(a)] are 30\% smaller than the unweighted ones, if compared with the entire illuminated area (Fig. 4). Qualitatively, however, the trends are nearly the same: in the thermal-dent region, the slope errors in the meridional and sagittal planes are close to each other. The minimal meridional and sagittal errors are still nearly the same.

The evolution of the calculated meridional slope errors with absorbed power is in good agreement with the experimental data (Chumakov et al., 2014), see Fig. 5(a). The value of the minimal calculated meridional slope error $(1.140 \mu \mathrm{rad}$, Table 4) is in excellent agreement with the smallest slope error measured with the same $2 \mathrm{~mm} \times 1 \mathrm{~mm}$ primary slit in the experiment $[\sim 1.1 \mu \mathrm{rad}$, Fig. $6(a)$ in Chumakov et al. (2014)].
Fig. 5(b) shows the weighted slope errors of the crystal surface predicted for the new ESRF-EBS machine. The significantly smaller sagittal slope errors result from the smaller horizontal size of the central cone, i.e. a much narrower horizontal region of the crystal surface 'probed' by monochromatic beam. In corresponding optimal conditions, the minimal sagittal error is smaller than the minimal meridional error by a factor of $\sim 2.7$. Both surface shapes corresponding to minimal sagittal slope error (at $\sim 162.5 \mathrm{~mA}$ ring current) and minimal meridional slope error $(\sim 185 \mathrm{~mA})$ are shown in Figs. 3(b) and 3(c), respectively.

\subsection{Angular distortions of the reflected beam}

Figs. 5(c) and 5(d) show the angular distortions of the reflected radiation analytically calculated from the slope errors using equations (1) and (2) and directly modelled by ray tracing using the initial height profiles of the crystal surface.

The results of the two approaches are in perfect agreement with each other. As expected from equations (1) and (2) and as confirmed by ray tracing, the sagittal distortions are reduced by the 'forgiveness' factor of $\sin \left(\theta_{\mathrm{B}}\right)$. In corresponding optimal conditions, the sagittal angular distortions are smaller than the meridional ones by a factor of $\sim 6.5$ for ESRF- 1 [Fig. 5(c)].

For the new EBS machine the sagittal angular distortions of the reflected beam [Fig. $5(d)]$ are smaller than the meridional ones by a factor of $\sim 20$, decreasing down to $\sim 0.1 \mu \mathrm{rad}$ (see Table 4). 
4.3. Performance of an HHLM with horizontal scattering plane at the EBS machine

The converging results of the FEA and ray tracing show that, for the EBS machine, the sagittal angular distortions of the reflected beam are expected to be smaller than the meridional ones by more than an order of magnitude. When a highquality beam is required specifically in the vertical plane, the obtained results provide strong arguments to use high-heatload monochromators with horizontal scattering plane. The vanishing distortions in the sagittal direction will allow for preserving high quality of the wavefront in the vertical plane for downstream optics. The higher distortions in the meridional plane will affect the horizontal properties of the beam, not critical for downstream high-resolution optics.

In order to evaluate the advantages of a high-heat-load monochromator with horizontal scattering plane, one should compare the residual sagittal angular distortions of the reflected beam with the angular size of the beam source. The angular divergence of a point source can be eliminated by collimating optics, e.g. by compound refractive lenses (CRLs) to practically zero. However, the angular size of an extended source (as seen at the position of the CRLs) is the only bottleneck which cannot be overcome and, therefore, this is the parameter which has to be compared with the angular distortions of the HHLM crystals (as stated above, possible corrections of the heat-load distortions by dedicated optics are beyond the scope of this paper).

As discussed above, the expected residual distortions of the reflected beam in the sagittal plane are about $0.1 \mu \mathrm{rad}$ (see Table 4). This is considerably smaller than the vertical angular ${ }^{6}$ source size of $\sim 0.42 \mu \mathrm{rad}$ for multibunch modes (see Table 1).

For timing modes, where the limited storage ring current ( $~ 90 \mathrm{~mA}$ ) does not allow to heat the silicon crystal to the optimal conditions, the residual distortions of the reflected beam in the sagittal direction are still small, about $0.5 \mu \mathrm{rad}$ [Fig. $5(d)$ ]. This is considerably smaller than the vertical angular source size of $\sim 0.93 \mu \mathrm{rad}$ for timing modes (see Table 1). These results identify the HHLM with horizontal scattering plane as a device essentially free of the heat-load effects in the vertical plane, both for multibunch and timing modes.

One also has to evaluate potentially negative aspects of the horizontal scattering plane. First of all, these are possible losses of beam intensity caused by the still slightly larger horizontal divergence and by the slightly smaller angular acceptance of the monochromator in the horizontal scattering plane (reduced by the polarization factor).

For the EBS-ESRF machine (see Table 1), in non-timing modes the horizontal and vertical divergences are about $14 \mu \mathrm{rad}$ and $10 \mu \mathrm{rad}$, respectively. In timing modes, they are even closer, about $14.2 \mu \mathrm{rad}$ and $13.6 \mu \mathrm{rad}$. Thus, the differences between horizontal and vertical divergences are not crucial.

The polarization factor $P=\cos \left(2 \theta_{\mathrm{B}}\right)=0.962$ reduces the angular acceptance of the $\mathrm{Si}(111)$ reflection from $18.3 \mu \mathrm{rad}$

${ }^{6} \mathrm{We}$ assume a CRL-source distance of $31 \mathrm{~m}$. in the vertical scattering plane to $17.7 \mu \mathrm{rad}$ in the horizontal scattering plane. This is still larger than the horizontal divergence of $14.2 \mu \mathrm{rad}$ of the new EBS machine. Therefore, the losses are tolerable: according to Table 2, switching from the vertical to the horizontal scattering plane decreases the peak throughput of monochromatic radiation $T_{\mathrm{M}}$ by $11.5 \%$ for non-timing modes $\left(\epsilon_{\mathrm{v}}=5 \mathrm{pm}\right)$ and by $3.3 \%$ for timing modes $\left(\epsilon_{\mathrm{v}}=50 \mathrm{pm}\right)$.

Finally, one has to consider that the optimal heat conditions for the minimal sagittal slope errors require a somewhat smaller absorbed heat load than for the meridional ones, namely $\sim 300 \mathrm{~W}$ instead of $\sim 340 \mathrm{~W}$ [see Table 4 and Fig. $5(d)$ ]. For equal storage ring current, this reduction of the heat load can be achieved by a slight closing of the primary slit from $2.0 \mathrm{~mm} \times 1.0 \mathrm{~mm}$ to a slightly smaller $1.88 \mathrm{~mm} \times 0.94 \mathrm{~mm}$ aperture.

This will still allow for accepting the entire central cone of monochromatic radiation (measured recently at ID18, $0.40 \mathrm{~mm} \times 0.38 \mathrm{~mm}, \mathrm{FWHM})$.

\section{Summary and conclusions}

In essence, this study shows that the high-heat-load monochromator and sensitive downstream optics should work not in the same but in perpendicular scattering planes. For the EBS machine, this allows for the reduction of the angular distortions of the X-ray beam incident on the downstream optics by an order of magnitude or so. Thus, if the sensitive downstream optics must work in the vertical scattering plane, the HHLM should be designed with horizontal scattering plane, and vice versa. When the choice of the scattering plane for downstream optics is arbitrary, the horizontally scattering monochromator is still preferable, as even with the EBS machine the quality of the beam in the vertical plane is superior than in the horizontal one, and this quality deserves to be preserved.

For the specific case considered here, the ESRF-EBS storage ring and the double-crystal $\mathrm{Si}(111)$ cryogenically cooled monochromator for X-rays with energy of $14.412 \mathrm{keV}$, switching the scattering plane from vertical to horizontal leads to negligible losses of intensity, but reduces the vertical angular distortions below the angular size of the beam source. This qualifies the considered monochromator as a device essentially free of the heat-load effects in the vertical plane.

\section{APPENDIX $A$ \\ Algebraic derivation of equations (1) and (2)}

The following calculations use a coordinate system with $\hat{y}$ (left seen along the beam axis) and $\hat{z}$ (normal to surface). The scattering plane is the $x z$ plane, i.e. the centre of the incident beam is in the $x z$ plane, inclined by $\theta_{\mathrm{B}}$ relative to the $\hat{x}$-axis (see Fig. 6). Consequently, meridional slope errors concern the $x z$ plane, and sagittal slope errors concern the $y z$ plane. 


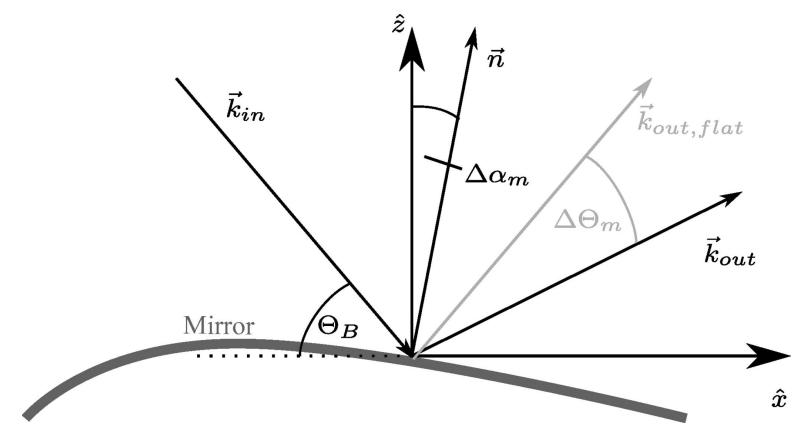

Figure 6

Illustration of the incident beam in two dimensions to show the effect of a slope error $\Delta \alpha_{\mathrm{m}}$ in the meridional direction on the incoming beam $\mathbf{k}_{\text {in }}$.

\section{A1. Flat surface}

Assume a beam travelling in the direction $\mathbf{k}_{\text {in }}$. The beam impinges on the mirror at the centre of the coordinate system, forming an angle $\theta_{\mathrm{B}}$ with the mirror surface at the centre of the mirror (see the illustration in Fig. 6),

$$
\mathbf{k}_{\text {in }}=\left(\begin{array}{c}
\cos \left(\theta_{\mathrm{B}}\right) \\
0 \\
-\sin \left(\theta_{\mathrm{B}}\right)
\end{array}\right) .
$$

Since we consider a purely geometic construction, the wavelength and the length of $\mathbf{k}_{\text {in }}$ do not matter.

The direction of the reflected beam is found using the usual formula

$$
\mathbf{k}_{\text {out }}=\mathbf{k}_{\text {in }}-2 \frac{\mathbf{k}_{\text {in }} \cdot \mathbf{n}}{|\mathbf{n}|^{2}} \mathbf{n},
$$

where for a perfectly flat mirror the surface normal $\mathbf{n}=\hat{z}$ is constant and does not depend on the position on the mirror. This then yields the nominal reflected beam

$$
\mathbf{k}_{\text {out }, \text { flat }}=\left(\begin{array}{c}
\cos \left(\theta_{\mathrm{B}}\right) \\
0 \\
\sin \left(\theta_{\mathrm{B}}\right)
\end{array}\right) .
$$

The beams reflected of a surface affected by slope errors will be compared with this nominal reflected beam. In particular, angular deviations $\Delta \theta_{\mathrm{m}, \mathrm{s}}$ from this direction are found by projecting the aberrated beam direction $\mathbf{k}_{\text {out }}$ onto unit vectors $\hat{k}_{\mathrm{m}, \mathrm{s}}$ in the meridional and sagittal planes that are perpendicular to this nominal reflected beam, $\mathbf{k}_{\text {out,flat }}$,

$$
\begin{gathered}
\hat{k}_{\mathrm{m}}=\left(\begin{array}{c}
\sin \left(\theta_{\mathrm{B}}\right) \\
0 \\
-\cos \left(\theta_{\mathrm{B}}\right)
\end{array}\right) . \\
\hat{k}_{\mathrm{s}}=\left(\begin{array}{l}
0 \\
1 \\
0
\end{array}\right), \\
\Delta \theta_{\mathrm{m}}=\mathbf{k}_{\text {out }} \cdot \hat{k}_{\mathrm{m}}, \\
\Delta \theta_{\mathrm{s}}=\mathbf{k}_{\text {out }} \cdot \hat{k}_{\mathrm{s}} .
\end{gathered}
$$

\section{A2. Slope errors}

We assume that the diffraction from a crystal can be treated as a specular reflection of a mirror surface centred at the origin of the coordinate system. Let the mirror's surface be defined by the function $z(x, y)$.

In the presence of slope errors $\Delta \alpha_{\mathrm{m}}=\partial z / \partial x$ and $\Delta \alpha_{\mathrm{s}}=$ $\partial z / \partial y$ in the sagittal and meridional direction, the surface normal is given by

$$
\mathbf{n}\left(\Delta \theta_{\mathrm{m}}, \Delta \theta_{\mathrm{s}}\right)=\left(\begin{array}{c}
\Delta \alpha_{\mathrm{m}} \\
\Delta \alpha_{\mathrm{s}} \\
1
\end{array}\right),
$$

with $\left|\mathbf{n}\left(\Delta \alpha_{\mathrm{m}}, \Delta \alpha_{\mathrm{s}}\right)\right| \simeq 1$ if $\Delta \alpha_{\mathrm{m}, \mathrm{s}} \ll 1$. Equation (4) then yields the aberrated direction of the reflected beam,

$$
\begin{aligned}
\mathbf{k}_{\text {out }}\left(\Delta \alpha_{\mathrm{m}}, \Delta \alpha_{\mathrm{s}}\right)= & \left(\begin{array}{c}
\cos \left(\theta_{\mathrm{B}}\right) \\
0 \\
-\sin \left(\theta_{\mathrm{B}}\right)
\end{array}\right) \\
& -2 \frac{\cos \left(\theta_{\mathrm{B}}\right) \Delta \alpha_{\mathrm{m}}-\sin \left(\theta_{\mathrm{B}}\right)}{1+\Delta \alpha_{\mathrm{m}}^{2}+\Delta \alpha_{\mathrm{s}}^{2}}\left(\begin{array}{c}
\Delta \alpha_{\mathrm{m}} \\
\Delta \alpha_{\mathrm{s}} \\
1
\end{array}\right) \\
\simeq & \left(\begin{array}{c}
\cos \left(\theta_{\mathrm{B}}\right)+2 \sin \left(\theta_{\mathrm{B}}\right) \Delta \alpha_{\mathrm{m}} \\
2 \sin \left(\theta_{\mathrm{B}}\right) \Delta \alpha_{\mathrm{s}} \\
\sin \left(\theta_{\mathrm{B}}\right)-2 \cos \left(\theta_{\mathrm{B}}\right) \Delta \alpha_{\mathrm{m}}
\end{array}\right) .
\end{aligned}
$$

The corresponding angular errors [equations (6) to (9)] are

$$
\begin{gathered}
\Delta \theta_{\mathrm{m}}=2 \Delta \alpha_{\mathrm{m}}, \\
\Delta \theta_{\mathrm{s}}=2 \Delta \alpha_{\mathrm{s}} \sin \left(\theta_{\mathrm{B}}\right),
\end{gathered}
$$

i.e. the relations given in equations (1) and (2).

\section{APPENDIX $B$}

\section{Geometrical derivation of equations (1) and (2)}

The fundamental impact of macroscopic surface deformations on the beam propagation after reflection can be illustrated with a simplified geometrical approach. Consider a beam with cross section $S_{\mathrm{m}}$ and $S_{\mathrm{s}}$ impinging under an angle $\theta_{\mathrm{B}}$ on a surface with radii $R_{\mathrm{m}}$ and $R_{\mathrm{s}}$. Upon reflection along the meridional direction the beam is projected over a footprint of

$$
L_{\mathrm{m}} \simeq \frac{S_{\mathrm{m}}}{\sin \left(\theta_{\mathrm{B}}\right)} .
$$

The corresponding surface slope variation is given by

$$
\Delta \alpha_{\mathrm{m}}=\frac{L_{\mathrm{m}}}{R_{\mathrm{m}}}=\frac{S_{\mathrm{m}}}{R_{\mathrm{m}} \sin \left(\theta_{\mathrm{B}}\right)} .
$$

The total angular spread of the reflected beam is then

$$
\Delta \theta_{\mathrm{m}}=2 \Delta \alpha_{\mathrm{m}}=2 \frac{S_{\mathrm{m}}}{R_{\mathrm{m}} \sin \left(\theta_{\mathrm{B}}\right)} .
$$

Along the sagittal direction the footprint is not affected by the angle of incidence and 


$$
\Delta \alpha_{\mathrm{s}}=\frac{S_{\mathrm{s}}}{R_{\mathrm{s}}} .
$$

After reflection only the normal component $k_{y}=k_{0} \sin \left(\theta_{\mathrm{B}}\right)$ of the wavevector is tilted. Over the full sagittal beam section this tilt spreads over

$$
\Delta \theta_{\mathrm{s}}=2 \Delta \alpha_{\mathrm{s}} \frac{k_{y}}{k_{0}}=2 \frac{S_{\mathrm{s}} \sin \left(\theta_{\mathrm{B}}\right)}{R_{\mathrm{s}}} .
$$

A comparison of the two cases returns

$$
\frac{\Delta \theta_{\mathrm{m}}}{\Delta \theta_{\mathrm{s}}}=\frac{S_{\mathrm{m}}}{R_{\mathrm{m}}} \frac{R_{\mathrm{s}}}{S_{\mathrm{s}}} \frac{1}{\sin ^{2}\left(\theta_{\mathrm{B}}\right)} .
$$

In the simple case of a spherical surface $\left(R_{\mathrm{s}}=R_{\mathrm{m}}=R\right)$ and a symmetric beam $\left(S_{\mathrm{s}}=S_{\mathrm{m}}\right)$ this equation simplifies to

$$
\frac{\Delta \theta_{\mathrm{m}}}{\Delta \theta_{\mathrm{s}}}=\frac{1}{\sin ^{2}\left(\theta_{\mathrm{B}}\right)} \text {. }
$$

The same result is obtained by comparing the inverse focal distances of both cases,

$$
\frac{f_{\mathrm{s}}}{f_{\mathrm{m}}}=\frac{R /\left[2 \sin \left(\theta_{\mathrm{B}}\right)\right]}{\left[R \sin \left(\theta_{\mathrm{B}}\right)\right] / 2}=\frac{1}{\sin ^{2}\left(\theta_{\mathrm{B}}\right)} .
$$

At small-angle incidence, however, for a symmetric beam the radiation footprint on the crystal surface is by a factor of $1 / \sin \left(\theta_{\mathrm{B}}\right)$ longer in the meridional direction than in the sagittal one. This makes the meridional radius $R_{\mathrm{m}}$ significantly larger than the sagittal $R_{\mathrm{s}}$, approximately by the same factor. Therefore, the meridional and sagittal slope errors [defined by equations (15) and (17)] are close to each other (see Fig. 4) and the ratio of the angular distortions $\Delta \theta_{\mathrm{m}} / \Delta \theta_{\mathrm{s}}$ is close to $1 / \sin \left(\theta_{\mathrm{B}}\right)$ [see Figs. 5(c) and $5(d)$ ].

\section{APPENDIX $C$}

\section{C1. Finite-element analysis}

C1.1. Surface deposition of the heat load and mirror reflection of X-rays. The depth-distributed absorption of $\mathrm{X}$-rays in the crystal was approximated by surface deposition of the heat load. This simplification was validated by comparing the length scales of the volumetric absorption (absorbed power per volume) $p_{\mathrm{v}}(z)$ of the considered insertion device radiation with the expected temperature profile $T(z)$ in the crystal with $z$ being the in-depth coordinate perpendicular to the substrate surface. This comparison is illustrated in Fig. 7.

The volumetric absorption $p_{\mathrm{v}}(z)$ was calculated with the help of the synchrotron radiation code XOP (Sanchez del Rio \& Dejus, 2011) included in the package OASYS (Rebuffi \& Sanchez del Rio, 2017). First, the corresponding insertion device spectrum was calculated at a distance of $31 \mathrm{~m}$ from the source through the primary slit aperture $A_{\perp}=2.22 \mathrm{~mm} \times$ $1.11 \mathrm{~mm}$. Next, we calculated the power $P\left(z_{\perp}\right)$ absorbed in uniform blocks of Si with thickness $z_{\perp}$, oriented perpendicular to the beam. For this we used the XOP power module for different values of $z_{\perp}$ with logarithmic spacing. The volumetric

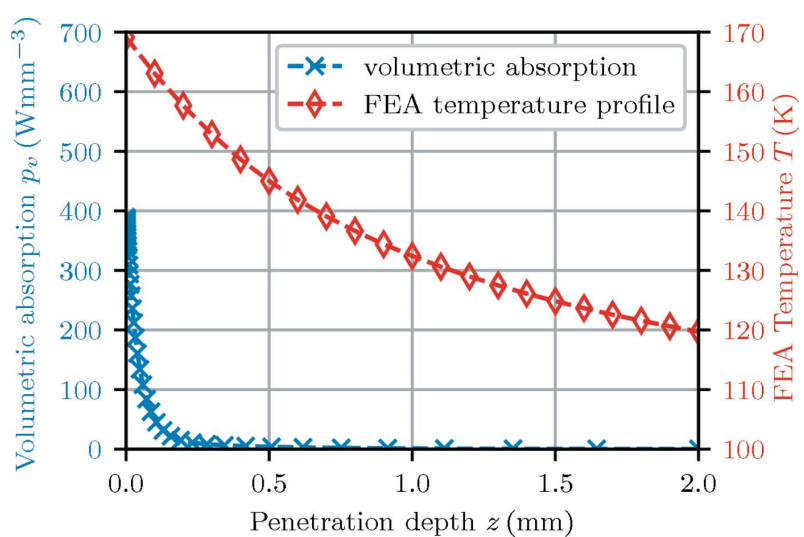

Figure 7

Validation of the assumption of surface absorption in the FEA model. Comparison of the length scales of: (blue) volumetric absorption $p_{\mathrm{v}}(z)$ of a U20 undulator (period $20 \mathrm{~mm}$, magnetic length $4.8 \mathrm{~m}$, gap $11 \mathrm{~mm}$ ) in Si at a Bragg angle of $\theta_{\mathrm{B}}=7.88^{\circ}$ with $z$ perpendicular to the surface and (red) expected temperature profile calculated by FEA.

power (power density) is the derivative of $P\left(z_{\perp}\right)$, normalized to the illuminated area $A_{\perp}$,

$$
p_{\mathrm{v}, \perp}\left(z_{\perp}\right)=\frac{1}{A} \frac{\partial P\left(z_{\perp}\right)}{\partial z_{\perp}} .
$$

Finally, the $z$-axis of the resulting volumetric power curve and the illuminated area were scaled to account for the grazingincidence angle, $\theta_{\mathrm{B}}=7.88^{\circ}$,

$$
\begin{gathered}
z=\sin \left(\theta_{\mathrm{B}}\right) z_{\perp}, \\
A=\frac{A_{\perp}}{\sin \left(\theta_{\mathrm{B}}\right)}, \\
p_{\mathrm{v}}(z)=\sin \left(\theta_{\mathrm{B}}\right) p_{\mathrm{v}, \perp}\left[\frac{z}{\sin \left(\theta_{\mathrm{B}}\right)}\right] .
\end{gathered}
$$

The temperature profile $T(z)$ was determined with ANSYS. The diamond-shaped markers illustrate the approximate mesh size (edge length $0.1 \mathrm{~mm}$ ). The temperature gradient at $z=0$ corresponds to the expected thermal gradient,

$$
\begin{aligned}
\frac{\mathrm{d} T}{\mathrm{~d} z}(z=0) & =\frac{-q_{z}(0)}{k(T=150 \mathrm{~K})} \\
& \simeq \frac{27 \mathrm{~W} \mathrm{~mm}^{-2}}{400 \mathrm{~W} \mathrm{~m}^{-1} \mathrm{~K}^{-1}}=67.5 \mathrm{~K} \mathrm{~mm}^{-1}
\end{aligned}
$$

with $-q_{z}(0)$ as the maximum thermal flux at the centre of the footprint and $k(T=150 \mathrm{~K})$ as the thermal conductivity of silicon at the expected approximate maximum temperature which was validated by FEA.

The corresponding length scales $z_{0}$ of $p_{\mathrm{v}}(z)$ and $T(z)$ are $\sim 0.04 \mathrm{~mm}$ and $\sim 1.2 \mathrm{~mm}$, respectively, assuming exponential decreases. Thus, the thermal conduction has a length scale approximately 30 times longer than the volumetric absorption. This justifies the assumption of surface absorption. Furthermore, the final mesh size used for the FEA model with an edge length of $L=0.1 \mathrm{~mm}$ at the surface with a growth factor of 1.2 sufficiently resolves the gradient as the edge length is significantly shorter than the corresponding length scale $L<$ 
$0.1 z_{0}[T(z)]$. The approximations are further validated by the reasonable agreement between the simulations and the experimental values.

The distortion of the crystal is governed by the temperature profile. Therefore the length scale of distortions is the length scale of the temperature variations, i.e. $\sim 1.2 \mathrm{~mm}$. The length scale of X-ray diffraction is the extinction depth, $\sim 1.5 \mu \mathrm{m}$, such that X-ray diffraction can be approximated by specular mirror reflection.

\section{C2. Gaussian-weighted standard deviation of slope errors}

Only X-rays of the correct photon energy are reflected by the monochromator. The EBS source is highly dispersive, and the monochromatic beam is narrower than the heat load profile. We therefore use the monochromatic intensity profile as the weighting function. The varying slope errors over the whole beam footprint $\Delta \alpha_{\mathrm{m}}(x, y)$ and $\Delta \alpha_{\mathrm{s}}(x, y)$ (in the meridional and sagittal direction, respectively) can then be expressed in statistical terms as standard deviations $\sigma\left(\Delta \alpha_{\mathrm{m}}\right)$ and $\sigma\left(\Delta \alpha_{\mathrm{s}}\right)$.

These standard deviations over the beam footprint (here the $x-y$-plane) are calculated via the variance $\sigma^{2}$ for a $2 \mathrm{D}$ function,

$$
\sigma_{\mathrm{m}, \mathrm{s}}^{2}=\frac{\int_{A} w(x, y)\left[f_{\mathrm{m}, \mathrm{s}}(x, y)-\bar{f}_{\mathrm{m}, \mathrm{s}}\right]^{2} \mathrm{~d} x \mathrm{~d} y}{\int_{A} w(x, y) \mathrm{d} x \mathrm{~d} y},
$$

where $w(x, y)$ denotes the considered weighting function. $\bar{f}_{\mathrm{m}, \mathrm{s}}=$ $\int_{A} w(x, y) f_{\mathrm{m}, \mathrm{s}}(x, y) \mathrm{d} x \mathrm{~d} y / \int_{A} w(x, y) \mathrm{d} x \mathrm{~d} y$ is the average slope, and $A$ is the illuminated surface area of the crystal. Here, coordinates $x$ and $y$ are in the beam and left directions, respectively. In this context, the weighting function $w(x, y)$ is the Gaussian intensity distribution of photons of the considered energy $14.412 \mathrm{keV}$. Hence it is defined as

$$
w(x, y)=\frac{2.355^{2}}{\left(4 \pi^{2} L_{\mathrm{m}}^{2} L_{\mathrm{s}}^{2}\right)^{1 / 2}} \exp \left[-2.355^{2}\left(\frac{x^{2}}{2 L_{\mathrm{m}}^{2}}+\frac{y^{2}}{2 L_{\mathrm{s}}^{2}}\right)\right]
$$

with $L_{\mathrm{s}}$ and $L_{\mathrm{m}}$ as beam sizes in terms of full width at halfmaximum (FWHM) in the meridional and sagittal direction projected on the mirror. The use of FWHM requires the introduction of $2.355^{2}$ terms for conversions to corresponding standard deviations. Considering the vertical deflection situation $L_{\mathrm{s}}$ and $L_{\mathrm{m}}$ result from the beam sizes in the vertical and horizontal direction by

$$
\begin{gathered}
L_{\mathrm{m}}=\frac{1}{\sin (\theta)} S_{\mathrm{m}}, \\
L_{\mathrm{s}}=S_{\mathrm{s}},
\end{gathered}
$$

relevant values for the paper for beam sizes $S_{\mathrm{m}}$ and $S_{\mathrm{s}}$ are given in Table 3. Equation (29) corresponds to the same assumption made in equation (14) in Appendix $B$.

The function $f_{\mathrm{m}, \mathrm{s}}(x, y)$ in equation (27) is the function to be weighted and represents here the slopes of the finite-elementcalculated height profile $z(x, y)$ of the footprint as derivatives with respect to the footprint coordinates,

$$
\begin{gathered}
f_{\mathrm{m}}(x, y)=\Delta \alpha_{\mathrm{m}}=\frac{\partial z(x, y)}{\partial x} \quad \text { (meridional), } \\
f_{\mathrm{s}}(x, y)=\Delta \alpha_{\mathrm{s}}=\frac{\partial z(x, y)}{\partial y} \quad \text { (sagittal) }
\end{gathered}
$$

depending on which slope errors are to be evaluated. To evaluate the integral in (27) one can use numerical approximations such as a Riemann sum or trapezoidal rule in the post-processing of the FEA results. Another option is to use methods already implemented in the Python programming language, e.g. numpy.average from the numpy package. The method numpy.gradient can be used to calculate the partial derivatives from equations (31) and (32). This option was chosen in the work for this project.

Evaluating equation (27) with slope errors defined as in (31) or (32) yields the standard deviation of the slope errors,

$$
\sigma\left(\Delta \alpha_{\mathrm{m}, \mathrm{s}}\right)=\sqrt{\sigma_{\mathrm{m}, \mathrm{s}}^{2}}
$$

over the whole footprint in the direction of interest. These slope errors $\Delta \alpha_{\mathrm{m}}$ and $\Delta \alpha_{\mathrm{s}}$ correspond to the way they are used in both equations (1) and (2).

\section{Acknowledgements}

We are grateful to the ESRF Accelerator and Source division for the stable operation of the new EBS machine during the test mode in March 2020, enabling the verification of the new source parameters. AIC thanks J. Chavanne and R. Versteegen for discussion of the parameters of the EBS machine. The authors thank Friederike Ewald for the helpful discussions on the calculation of source size and divergence.

\section{References}

Biasci, J., Bouteille, J., Carmignani, N., Chavanne, J., Coulon, D., Dabin, Y., Ewald, F., Farvacque, L., Goirand, L., Hahn, M., Jacob, J., LeBec, G., Liuzzo, S., Nash, B., Pedroso-Marques, H., Perron, T., Plouviez, E., Raimondi, P., Revol, J., Scheidt, K. \& Serrière, V. (2014). Synchrotron Radiat. News, 27(6), 8-12.

Carpentier, P., Rossat, M., Charrault, P., Joly, J., Pirocchi, M., Ferrer, J.-L., Kaikati, O. \& Roth, M. (2001). Nucl. Instrum. Methods Phys. Res. A, 456, 163-176.

Castro, A. R. B. de \& Reininger, R. (1991). Nucl. Instrum. Methods Phys. Res. A, 307, 135-141.

Chavanne, J., Le Bec, G. \& Penel, C. (2011). Synchrotron Radiat. News, 24(3), 10-13.

Chumakov, A., Rüffer, R., Leupold, O., Celse, J.-P., Martel, K., Rossat, M. \& Lee, W.-K. (2004). J. Synchrotron Rad. 11, 132-141.

Chumakov, A. I., Sergeev, I., Celse, J.-P., Rüffer, R., Lesourd, M., Zhang, L. \& Sanchez del Rio, M. (2014). J. Synchrotron Rad. 21, 315-324.

Chumakov, A. I., Shvyd'ko, Y., Sergueev, I., Bessas, D. \& Rüffer, R. (2019). Phys. Rev. Lett. 123, 097402.

DiGennaro, R., Gee, B., Guigli, J., Hogrefe, H., Howells, M. \& Rarback, H. (1988). Nucl. Instrum. Methods Phys. Res. A, 266, 498 506.

Lee, W.-K., Fernandez, P. \& Mills, D. M. (2000). J. Synchrotron Rad. 7, $12-17$.

Lee, W.-K., Fezzaa, K., Fernandez, P., Tajiri, G. \& Mills, D. M. (2001). J. Synchrotron Rad. 8, 22-25.

Middelmann, T., Walkov, A., Bartl, G. \& Schödel, R. (2015). Phys. Rev. B, 92, 174113 . 
Onuki, H. \& Elleaume, P. (2003). Editors. Undulators, Wigglers, and their Applications, pp. 75-79. London/New York: Taylor \& Francis.

Raimondi, P. (2016). Proceedings of the Seventh International Particle Accelerator Conference (IPAC'16), 8-13 May 2016, Busan, Korea, pp. 2023-2027.

Rebuffi, L. \& Sanchez del Rio, M. (2016). J. Synchrotron Rad. 23, 1357-1367.

Rebuffi, L. \& Sanchez del Rio, M. (2017). Advances in Computational Methods for X-ray Optics IV, edited by K. Sawhney \& O. Chubar, p. 28. SPIE.

Rüffer, R. \& Chumakov, A. I. (1996). Hyperfine Interact. 97-98, 589604.

Sanchez del Rio, M., Canestrari, N., Jiang, F. \& Cerrina, F. (2011). J. Synchrotron Rad. 18, 708-716.

Sanchez del Rio, M. \& Dejus, R. J. (2011). Proc. SPIE, 8141, 814115.
Sanchez del Rio, M., Perez-Bocanegra, N., Shi, X., Honkimäki, V. \& Zhang, L. (2015). J. Appl. Cryst. 48, 477-491.

Tajiri, G., Lee, W.-K., Fernandez, P., Mills, D. M., Assoufid, L. \& Amirouche, F. (2001). J. Synchrotron Rad. 8, 1140-1148.

Tanaka, T. \& Kitamura, H. (2009). J. Synchrotron Rad. 16, 380-386.

Touloukian, Y., Powell, R., Ho, C. \& Klemens, P. (1971). Thermophysical Properties of Matter, Vol. 1, Thermal Conductivity Metallic Elements and Alloys. Defense Technical Information Center.

Zhang, L., Barrett, R., Cloetens, P., Detlefs, C. \& Sanchez del Rio, M. (2014). J. Synchrotron Rad. 21, 507-517.

Zhang, L., Lee, W.-K., Wulff, M. \& Eybert, L. (2003). J. Synchrotron Rad. 10, 313-319.

Zhang, L., Sanchez del Rio, M., Monaco, G., Detlefs, C., Roth, T., Chumakov, A. I. \& Glatzel, P. (2013). J. Synchrotron Rad. 20, $567-$ 580 . 